\title{
Different roles of surveillance positron emission tomography according to the histologic subtype of non-Hodgkin's lymphoma
}

Yu Ri Kim ${ }^{1}$, Soo-Jeong Kim² ${ }^{2}$ June-Won Cheong ${ }^{2}$, Yundeok Kim ${ }^{1}$, Ji Eun Jang ${ }^{2}$, Hyunsoo Cho ${ }^{2}$, Haerim Chung ${ }^{2}$, Yoo Hong Min ${ }^{2}$, Woo Ick Yang ${ }^{3}$, Arthur Cho ${ }^{4}$, and Jin Seok Kim²

${ }^{1}$ Division of Hematology, Department of Internal Medicine, Gangnam Severance Hospital, Yonsei University College of Medicine, Seoul; ${ }^{2}$ Division of Hematology, Department of Internal Medicine, Departments of ${ }^{3}$ Pathology and ${ }^{4}$ Nuclear Medicine, Severance Hospital, Yonsei University College of Medicine, Seoul, Korea

Received: November 6, 2019

Revised : January 3, 2020

Accepted: January 7, 2020

\section{Correspondence to}

Jin Seok Kim, M.D.

Division of Hematology,

Department of Internal

Medicine, Severance Hospital,

Yonsei University College

of Medicine, 50-1 Yonsei-ro,

Seodaemun-gu, Seoul 03722,

Korea

Tel: $+82-2-2228-1972$

Fax: +82-2-393-6884

E-mail: hemakim@yuhs.ac https://orcid.org/0000-0001$8986-8436$
Background/Aims: Although the use of surveillance ${ }^{18} \mathrm{~F}$-fluorodeoxyglucose (FDG) positron emission tomography/computed tomography (PET/CT) is discouraged in patients with diffuse large B-cell lymphoma, its usefulness in different subtypes has not been thoroughly investigated.

Methods: We retrospectively evaluated 157 patients who showed positive results on surveillance FDG-PET/CT every 6 months following complete response for up to 5 years. All of the patients also underwent biopsies.

Results: Seventy-eight (49.6\%) of 157 patients had true positive results; the remaining 79 (50.3\%), including eight (5.1\%) with secondary malignancies, were confirmed to yield false positive results. Among the 78 patients with true positive results, the disease in seven (8.9\%) had transformed to a different subtype. The positive predictive value (PPV) of FDG-PET/CT for aggressive B-cell non-Hodgkin's lympho$\mathrm{ma}(\mathrm{NHL})$ was lower than that for indolent B-cell or aggressive T-cell NHL $(p=0.003$ and $p=0.018$, respectively), especially in patients with a low/low-intermediate international prognostic index (IPI) upon a positive PET/CT finding. On the other hand, indolent B-cell and aggressive T-cell NHL patients showed PPVs of $>60 \%$, including those with low/low-intermediate secondary IPIs.

Conclusions: The role of FDG-PET/CT surveillance is limited, and differs according to the lymphoma subtype. FDG-PET/CT may be useful in detecting early relapse in patients with aggressive T-cell NHL, including those with low/low-intermediate risk secondary IPI; as already known, FDG-PET/CT has no role in aggressive B-cell NHL. Repeat biopsy should be performed to discriminate relapse or transformation from false positive findings in patients with positive surveillance FDG-PET/CT results.

Keywords: Positron emission tomography computed tomography; Sensitivity and specificity

\section{INTRODUCTION}

Positron emission tomography (PET) alone or combined with computed tomography (PET/CT) using flu- orine ${ }^{18} \mathrm{~F}$-fluorodeoxyglucose (FDG) is a highly sensitive and non-invasive imaging modality that is widely used for patients with malignant lymphoma. It is an essential imaging tool for evaluating the extent of disease at 
diagnosis, response assessment, and restaging of patients with FDG-avid lymphoma [1,2]. The International Working Group revised the response criteria for incorporating FDG-PET/CT in diffuse large B-cell lymphoma (DLBCL) and Hodgkin's lymphoma (HL) [2]. Although data acquired from interim FDG-PET/CT imaging are insufficient to modify treatment strategies, the utility of risk-adapted therapies based on such interim FDGPET/CT findings has been widely investigated $[3,4]$. On the other hand, the current recommendations discourage the use of FDG-PET/CT for surveillance [5]. Many studies on surveillance imaging found that using FDGPET-CT for routine follow-up did not reliably detect early relapse, especially in patients with curable lymphomas [6-8]. Routine radiologic imaging is associated with a number of drawbacks, including excessive cost, lead-time bias, cancer risk related to radiation exposure, and patient anxiety $[9,10]$. Moreover, FDG-PET/CT also produces a high false positive rate, which can lead to unnecessary biopsies [5,11].

Nevertheless, some studies suggested that surveillance FDG-PET/CT could be useful for a highly selected population of lymphoma. For example, elderly patients over 60 years or DLBCL patients with a baseline international prognostic index (IPI) $\geq 3$ in the first 18 months have been shown to benefit from surveillance FDGPET/CT [12,13]. Furthermore, different lymphoma subtypes carry varying prognoses in clinical practice, and relapsed lymphomas show different clinical courses according to disease aggressiveness. Aggressive non-Hodgkin's lymphoma (NHL) has a short relapse window, while indolent NHL has a long asymptomatic period before clinical relapse [7]. Early detection of relapse in patients with lower tumor burdens improves the chances of cure by salvage therapy [14]. Therefore, there may be a need to evaluate the role of FDG-PET/CT according to histologic subtypes. To date, few studies have assessed the role of surveillance FDG-PET/CT while considering different subtypes, especially T-cell lineage lymphoma. More detailed analyses that include lymphoma subtypes could help determine the usefulness of surveillance FDG-PET/CT in certain clinical situations.

Hence, the aim of this study was to evaluate the positive predictive value (PPV) of surveillance FDG-PET/CT according to histologic findings, and to analyze the rate of associated second malignancies or transformation to other subtypes. Moreover, we attempted to compare the benefits of surveillance FDG-PET/CT among patients with aggressive B-cell NHL, aggressive T-cell NHL, and indolent B-cell NHL.

\section{METHODS}

\section{Patients}

Data from 157 patients who were newly diagnosed with NHL between January 2003 and December 2013 at Severance Hospital were retrospectively analyzed. Their diagnoses were pathologically confirmed by hematology pathologists according to 2008 World Health Organization criteria [15]. During the study period, 1,700 patients were newly diagnosed with NHL, of whom 1,314 were treated at our institution; 971 patients achieved a complete response (CR) after first-line treatment. During the follow-up period, 157 patients showed positive findings on surveillance FDG-PET/CT within 5 years after achieving a CR, and all results were confirmed with biopsy at the suspicious site. We excluded patients with positive FDG-PET/CT findings discovered during unscheduled FDG-PET/CT scans, as well as patients whose diseases were not histologically confirmed. Upon diagnosis, patients underwent physical examination, FDG-PET/CT, bone marrow examination, and laboratory tests that included lactic dehydrogenase (LDH) measurement. The stage was assessed in accordance with the Ann Arbor staging system; lymphoma subtypes that do not use this staging system (such as cutaneous T-cell lymphoma) were excluded [16]. Each patient was stratified into one of four risk groups according to IPI criteria [17]. Secondary IPI was defined as IPI at the time of positive FDG-PET/CT findings. We classified lymphomas into indolent B-cell, aggressive B-cell, and aggressive T-cell NHLs [18]. Patients with lymphoma included 89 with DLBCL, 18 with marginal zone B-cell lymphoma, 20 with follicular lymphoma, eight with mantle cell lymphoma (MCL), five with Burkitt lymphoma, one with lymphoblastic lymphoma, two with extranodal natural killer/T (NK/T)-cell lymphoma-nasal type, and 14 with peripheral T-cell lymphoma. MCL was scored according to MCL IPI [19]. Six low risk patients were classified as having indolent B-cell NHL, while one patient with intermediate risk and another with high risk were 
Table 1. Patient characteristics

\begin{tabular}{lc}
\hline Variable & Value \\
\hline Age, yr (median) & $55.5(18-82)$ \\
Male sex & $91(58.0)$ \\
ECOG o-1 & $146(93)$ \\
Stage III/IV & $89(56.7)$ \\
Extranodal involvement $>1$ & $48(30.6)$ \\
Elevated LDH level & $69(43.9)$ \\
IPI & $74(47.1) / 33(21.0)$ \\
Low/low-intermediate & $36(22.9) / 14(8.9)$ \\
\hline High-intermediate/high & \\
\hline
\end{tabular}

Values are presented as median (range) or number (\%).

ECOG, Eastern Cooperative Oncology Group; LDH, lactic dehydrogenase; IPI, international prognostic index.

deemed to have aggressive B-cell NHL. This study was approved by the Institutional Review Board of Severance Hospital. Written informed consent by the patients was waived due to a retrospective nature of our study (IRB no. 3-2014-0289).

\section{Response assessment and ${ }^{18} \mathrm{~F}$-FDG-PET/CT}

Responses were assessed after treatment completion according to International Working Group criteria using FDG-PET/CT [2]. Surveillance FDG-PET/CT was performed at 6-month intervals in the first 2 years and every other year for up to 5 years. Patients with positive FDG-PET/CT findings underwent biopsies at FDG uptake sites. FDG-PET/CT results were reviewed visually by experienced nuclear medicine physicians. Positive scans were defined as the appearance of newly developed uptake relative to that in the mediastinal blood pool; negative scans were considered a lack of evidence of disease $[20,21]$. Patients with FDG uptake owing to suspected physiologic responses were not included in this study. The five-point visual assessment method was used to score FDG uptake [22]. Patients with Deauville scores of 1 or 2 were not included in this study, as these scores were interpreted as negative findings.

\section{Statistical methods}

We categorized patients according to true or false positive findings; PPV was defined as the proportion of true positive patients among those with positive surveillance FDG-PET/CT findings. "True positive" was defined as FDG uptake on FDG-PET/CT that was histologically confirmed as relapsed lymphoma, while "false positive" was defined as no evidence of recurred lymphoma on biopsy. The statistical significance of categorical variables was calculated using chi-squared tests. $p$ values < 0.05 were considered statistically significant in all tests. All statistical analyses were performed using SPSS for Windows version 23.0 (IBM Corp., Armonk, NY, USA).

\section{RESULTS}

\section{Patient characteristics}

During a median follow-up period of 47.0 months (range, 7 to 133), 1,186 FDG-PET/CT images were acquired, with 157 patients showing positive findings. Patient characteristics are summarized in Table 1. Among the 157 patients with positive FDG-PET/CT findings, 78 (49.7\%) were confirmed to have relapsed on biopsy, while 79 (50.3\%) had no evidence of relapse. PPVs of FDG-PT/ CT according to lymphoma aggressiveness are shown in Table 2. Among 971 patients, 90 patients diagnosed with relapse by symptoms and 69 patients underwent repeated biopsy. Relapse patients diagnosed by symptoms showed a higher PPV than those diagnosed by surveillance PET/CT in all subtypes (indolent B-cell NHL: $p=0.013$, aggressive B-cell NHL: $p<0.001$, and T-cell NHL: $p=0.048$ ).

Histological findings in the 79 false positive patients revealed eight secondary cancers (Table 3). FDG uptake in these patients was observed at extranodal sites that were distinct from the primary sites at diagnosis. The 
Table 2. Positive predictive values according to the aggressiveness of lymphoma and time from remission

\begin{tabular}{|c|c|c|c|c|c|c|c|c|c|}
\hline \multirow[b]{2}{*}{ Subtype } & \multirow[b]{2}{*}{$\begin{array}{c}\text { No. } \\
\text { (positive/ } \\
\text { total) }\end{array}$} & \multirow[b]{2}{*}{ PPV, \% } & \multirow[b]{2}{*}{$p$ value } & \multicolumn{3}{|c|}{ o-24 months } & \multicolumn{3}{|c|}{$24-60$ months } \\
\hline & & & & $\begin{array}{c}\text { No. } \\
\text { (positive/ } \\
\text { total) }\end{array}$ & $\mathrm{PPV}, \%$ & $p$ value & $\begin{array}{c}\text { No. } \\
\text { (positive/ } \\
\text { total) }\end{array}$ & PPV, \% & $p$ value \\
\hline Aggressive B-cell NHL & $37 / 96$ & 38.5 & & $27 / 78$ & 34.6 & & $10 / 18$ & 55.6 & \\
\hline vs. Indolent B-cell NHL & $29 / 44$ & 65.9 & 0.003 & $18 / 29$ & 62.1 & 0.015 & $11 / 15$ & $73 \cdot 3$ & 0.469 \\
\hline vs. Aggressive T-cell NHL & $12 / 17$ & 70.6 & 0.018 & $12 / 17$ & 70.6 & 0.013 & o/o & 0.0 & \\
\hline Total & $78 / 157$ & 49.6 & & & & & & & \\
\hline
\end{tabular}

PPV, positive predictive value; NHL, non-Hodgkin's lymphoma.

median age of these patients was 73 years (range, 57 to 79 ).

\section{PPVs according to lymphoma subtype}

Patient classification based on the subtype of lymphoma revealed that 44 patients had indolent B-cell NHL, 96 had aggressive B-cell NHL, and 17 had aggressive T-cell NHL. PPV of aggressive B-cell NHL (38.5\%) was significantly lower than those of indolent B-cell NHL (65.9\%) and aggressive T-cell NHL (70.6\%) ( $p=0.003$ and $p=0.018$, respectively) (Table 2 ). There was no difference in PPV according to rituximab treatment in patients with aggressive B-cell NHL $(p=0.999)$ or those with indolent B-cell NHL $(p=0.342)$. Analysis of durations since achieving CR showed that PPV of patients with aggressive B-cell NHL (34.6\%) was lower than that of patients with indolent B-cell NHL (62.1\%) or aggressive T-cell NHL (64.1\%) within 24 months $(p=0.015, p=0.013$, respectively), although the difference was not significant after 24 months $(p=0.469)$ (Table 2).

Among 78 patients with true positive results, lymphoma transformed into a different subtype in seven (8.9\%); four of these patients showed FDG uptake at sites other than the locations of their initial lesions, and five relapsed after 12 months of follow-up. These seven patients included four of 37 with aggressive B-cell NHL (10.8\%) and three of 29 with indolent B-cell NHL (10.3\%) with positive PPVs. None of the patients with aggressive T-cell NHL experienced subtype change (Table 4). High Deauville score was associated with high PPV. True positive and false positive rates were $26.1 \%$ and $73.9 \%$ in Deauville 3 group, $43.8 \%$ and $56.3 \%$ in Deauville 4 , and $59.3 \%$ and $40.7 \%$ in Deauville 5 group, respectively $(p=$ ०.011).

\section{PPV according to IPI at diagnosis or relapse}

There was no significant difference in PPVs between patients with low/low-intermediate risk and high-intermediate/high risk, as assessed by IPI at diagnoses ( $p$ $=0.734$ ). Moreover, PPVs did not differ when analyzed according to individual IPI components, such as age > 60 years $(p=0.327)$, Eastern Cooperative oncology Group (ECOG) performance status $\geq 2(p=0.765)$, stage $\geq$ III ( $p$ $=0.077)$, extranodal involvement at more than one site $(p=0.169)$, and elevated LDH levels $(p=0.631)$ at diagnosis. Among patients with low/low-intermediate risk IPIs at baseline, PPV of those with aggressive B-cell NHL (32.1\%) was lower than those with indolent B-cell NHL (62.2\%) and aggressive T-cell NHL (78.6\%) ( $p=0.006$ and $p=0.002$, respectively). However, there was no PPV difference according to subtype among patients with high-intermediate/high risk baseline IPI (Table 5).

At the time of performing FDG-PET/CT, 119 patients (75.8\%) were low risk, 25 (15.9\%) were low-intermediate risk, 11 (7.0\%) were high-intermediate risk, and two (1.3\%) were high risk according to secondary IPIs. Patients with low/low-intermediate risk secondary IPIs had lower PPVs than patients with high-intermediate/high risk secondary IPIs ( $45.8 \%$ vs. $92.3 \%, p=0.001$ ); this finding was clinically significant only in patients with aggressive B-cell NHL (34.1\% vs. 87.5\%, $p=0.005$ ). Among patients with low/low-intermediate risk secondary IPI, PPV of aggressive B-cell NHL was lower than those of indolent and aggressive T-cell NHLs $(p=0.001$ and $p=0.040$, respectively). PPVs of aggressive T-cell and indolent B-cell NHL patients were $64.3 \%$ each in those with low/low-intermediate risk IPI. There was no difference in PPV according to lymphoma subtypes among patients with high-intermediate/high risk secondary IPI (Table 5). 
Table 3. Histologic findings in false positive patients

\begin{tabular}{lcccc}
\hline Variable & Indolent B-cell NHL & Aggressive B-cell NHL & Aggressive T-cell NHL & Total, no. (\%) \\
\hline Second cancer & 1 & 7 & 0 & $8(10.1)$ \\
Inflammation/reactive & 13 & 43 & 3 & $59(74.9)$ \\
Normal & 1 & 9 & 2 & $12(15.2)$ \\
Total & 15 & 59 & 5 & 79 \\
\hline
\end{tabular}

NHL, non-Hodgkin's lymphoma.

Table 4. Patients with subtype changes

\begin{tabular}{lccc}
\hline $\begin{array}{l}\text { Lymphoma subtype } \\
\text { at diagnosis }\end{array}$ & $\begin{array}{c}\text { Primary site } \\
\text { at diagnosis }\end{array}$ & $\begin{array}{c}\text { Primary site } \\
\text { at relapse }\end{array}$ & $\begin{array}{c}\text { Lymphoma subtype } \\
\text { at relapse }\end{array}$ \\
\hline DLBCL & LN & LN & FL \\
DLBCL & LN & LN & FL \\
DLBCL & Oropharynx & Tonsil & HL \\
DLBCL & LN & Lung & MZBCL \\
FL & Soft tissue & LN & DLBCL \\
FL & LN & LN & DLBCL \\
MZBCL & Lung & Soft tissue & DLBCL \\
\hline
\end{tabular}

DLBCL, diffuse large B-cell lymphoma; LN, lymph node; FL, follicular lymphoma; HL, Hodgkin lymphoma; MZBCL, marginal zone B-cell lymphoma.

Table 5. Positive predictive values according to lymphoma subtypes and international prognostic index

\begin{tabular}{|c|c|c|c|c|c|c|}
\hline \multirow{2}{*}{ Variable } & \multicolumn{3}{|c|}{ Low/Low-intermediate } & \multicolumn{3}{|c|}{ High-intermediate/High } \\
\hline & No. (positive/total) & PPV & $p$ value & No. (positive/total) & PPV & $p$ value \\
\hline \multicolumn{7}{|l|}{ IPI at baseline } \\
\hline Aggressive B-cell NHL & $18 / 56$ & 32.1 & - & $19 / 40$ & 47.5 & - \\
\hline vs. Indolent NHL & $23 / 37$ & 62.2 & 0.006 & $6 / 7$ & $85 \cdot 7$ & 0.102 \\
\hline vs. Aggressive T-cell NHL & $11 / 14$ & 78.6 & 0.002 & $1 / 3$ & $33 \cdot 3$ & 0.999 \\
\hline \multicolumn{7}{|l|}{ IPI at relapse (secondary IPI) } \\
\hline Aggressive B-cell NHL & $30 / 88$ & 34.1 & - & $7 / 8$ & 87.5 & - \\
\hline vs. Indolent NHL & $27 / 42$ & $64 \cdot 3$ & 0.001 & $2 / 2$ & 100.0 & 0.999 \\
\hline vs. Aggressive T-cell NHL & $9 / 14$ & $64 \cdot 3$ & 0.040 & $3 / 3$ & 100.0 & 0.999 \\
\hline
\end{tabular}

PPV, positive predictive value; IPI, international prognostic index; NHL, non-Hodgkin's lymphoma.

\section{DISCUSSION}

We found that PPV of surveillance FDG-PET/CT is below $50 \%$, as more than half of the diagnoses turned out to be false positive when confirmed with biopsy. PPV of surveillance PET/CT was significantly lower than that of symptomatic relapse. This indicated that repeat biopsies should be considered for asymptomatic patients with FDG uptake on surveillance FDG-PET/CT to identify false positive results. Our false positive rate was relatively higher than in previous studies, as we included only results from asymptomatic patients with positive FDG-PET/CT results, and excluded data from patients with suspected clinical relapse. The most common histologic finding among the 79 patients with false positive results was inflammation or reactive hyperplasia; more- 
over, eight were diagnosed with secondary malignancies. High FDG uptake could help discriminate relapse from false positive results. Therefore, repeated biopsies would also serve to differentiate secondary malignancies from relapsed lymphoma or inflammation.

Notably, surveillance FDG-PET/CT had differing degrees of usefulness depending on the subtype of lymphoma. Although FDG-PET/CT for staging and response evaluation can provide confirmative evidence of DLBCL, it is not as useful for its surveillance. There are some reports about the usefulness of surveillance PET/ CT for highly selective patients. We hypothesized that the role of surveillance PET/CT would differ according to the subtype of lymphoma. In patients with aggressive B-cell NHL, surveillance FDG-PET/CT imaging was shown to have a limited role in predicting relapse, especially in patients with low/low-intermediate risk IPIs at diagnosis and at the time of relapse. Aggressive B-cell NHL showed statistically low PPV of surveillance FDGPET/CT within the first 24 months. After 24 months, PPV of surveillance FDG-PET/CT in patients with aggressive B-cell NHL was not different compared that of indolent lymphoma; this suggested that positive PET results should be interpreted carefully to discriminate the relapse.

One of the possible causes of the high false positive rate in aggressive B-cell NHL is suggested to be rituximab, which is known to have relatively long-lasting inflammatory changes associated with the recruitment of immune cells to the tumor $[23,24]$. However, only a proportion of patients with aggressive or indolent B-cell NHL were treated with a rituximab-containing regimen in our study, and there was no significant difference in the false positive rate between such patients and those who did not receive the drug. Therefore, false positivity in patients with aggressive B-cell NHL may be partially attributed to the nature of lymphoma itself, in addition to any effect of rituximab. Based on the current guidelines, evaluation of complete blood count, metabolic panels, and serum LDH every 3 months could be sufficient for patients with aggressive B-cell NHL.

PPV of surveillance FDG-PET/CT was relatively higher in patients with aggressive T-cell NHL than in those with aggressive B-cell NHL. In contrast to B-cell NHL, there are only a few existing studies of the role of FDG-PET/CT in T-cell NHL. Among patients with aggressive T-cell NHL in our study, 70\% showed true relapse based on FDG-PET/CT results. The benefit of surveillance FDG-PET/CT was similarly evident even in patients with secondary low/low-intermediate IPI. This finding suggested that surveillance FDG-PET/CT could be useful for detecting relapse before symptoms occur in patients with aggressive T-cell NHL. Most aggressive T-cell NHLs are considered FDG-avid; they have short progression-free survival rates and frequently relapse. Hence, early detection of relapse could provide the opportunity for timely salvage treatment.

Indolent lymphoma progresses slowly; most patients were diagnosed with relapse through follow-up imaging, rather than clinical signs or physical examination. In contrast, relapse of aggressive NHL was detected via clinical symptoms. PPV of FDG-PET/CT in patients with indolent NHL was relatively high, even in those with low/low-intermediate risk IPIs; although the detection of relapse in these patients does not necessarily require immediate treatment, transformation from indolent to aggressive NHL does require rapid detection and prompt intervention. Approximately 10\% of patients with true positive results exhibited histologic subtype changes. In patients with indolent B-cell NHL, detecting any transformation to aggressive lymphoma is much more important than discovering a relapse of indolent lymphoma. Currently, there is no evidence to prove that early treatment of indolent lymphoma prolongs survival, and routine FDG-PET/CT is not recommended for such patients. However, there remains a need to identify the most accurate lesion with which to detect any transformation using FDG-PET/CT; of course, repeat biopsies play an invaluable role as well.

Our study had some limitations. Given its retrospective, single-center nature, the enrollment of patients who received biopsies may have led to selection bias. There are many subtypes of lymphoma, and this study did not include enough patients to show a significance of difference subtypes. Still, all of the included patients underwent planned routine surveillance FDG-PET/CT at regular intervals. To specifically evaluate the role of routine surveillance FDG-PET/CT, patients with symptoms and signs of relapse were not included in this study. Moreover, we performed histologic confirmation of relapse via biopsy in all asymptomatic patients, and investigated the benefits of routine surveillance FDG- 
PET/CT in different lymphoma subtypes, as well as baseline or secondary IPI. Therefore, our findings will be useful for interpreting positive findings on routine surveillance FDG-PET/CT.

In conclusion, surveillance FDG-PET/CT produces varying PPVs according to the aggressiveness of lymphoma. While we confirmed that surveillance FDG$\mathrm{PET} / \mathrm{CT}$ is of little use for patients with aggressive B-cell NHL, there remains a need to investigate any benefit for FDG-PET/CT in patients with aggressive T-cell NHL. Importantly, histologic confirmation by repeat biopsies should be required to rule out false positive findings and distinguish true relapse from subtype transformation or secondary malignancies.

\section{KEY MESSAGE}

1. The role of ${ }^{18}$ F-fluorodeoxyglucose (FDG)- positron emission tomography/computed tomography (PET/CT) surveillance differs according to the subtypes of non-Hodgkin's lymphoma (NHL).

2. Surveillance FDG-PET/CT may be useful in patients with aggressive T-cell NHL.

3. Repeat biopsy should be recommended to detect relapse in NHL.

\section{Conflict of interest}

No potential conflict of interest relevant to this article was reported.

\section{REFERENCES}

1. Ansell SM, Armitage JO. Positron emission tomographic scans in lymphoma: convention and controversy. Mayo Clin Proc 2012;87:571-580.

2. Cheson BD, Pfistner B, Juweid ME, et al. Revised response criteria for malignant lymphoma. J Clin Oncol 2007;25:579-586.

3. Yang DH, Min JJ, Song HC, et al. Prognostic significance of interim 18F-FDG PET/CT after three or four cycles of R-CHOP chemotherapy in the treatment of diffuse large B-cell lymphoma. Eur J Cancer 2011;47:1312-1318.

4. Gallamini A, Kostakoglu L. Interim FDG-PET in Hod- gkin lymphoma: a compass for a safe navigation in clinical trials? Blood 2012;120:4913-4920.

5. Cheson BD, Fisher RI, Barrington SF, et al. Recommendations for initial evaluation, staging, and response assessment of Hodgkin and non-Hodgkin lymphoma: the Lugano classification. J Clin Oncol 2014;32:3059-3068.

6. Lee AI, Zuckerman DS, van den Abbeele AD, et al. Surveillance imaging of Hodgkin lymphoma patients in first remission: a clinical and economic analysis. Cancer 2010;116:3835-3842.

7. El-Galaly TC, Jakobsen LH, Hutchings M, et al. Routine imaging for diffuse large B-cell lymphoma in first complete remission does not improve post-treatment survival: a Danish-Swedish population-based study. J Clin Oncol 2015;33:3993-3998.

8. Jakobsen LH, Hutchings M, de Nully Brown P, et al. No survival benefit associated with routine surveillance imaging for Hodgkin lymphoma in first remission: a Danish-Swedish population-based observational study. Br J Haematol 2016;173:236-244.

9. Huang B, Law MW, Khong PL. Whole-body PET/CT scanning: estimation of radiation dose and cancer risk. Radiology 2009;251:166-174.

10. Barrington SF, Mikhaeel NG. When should FDG-PET be used in the modern management of lymphoma? $\mathrm{Br} \mathrm{J}$ Haematol 2014;164:315-328.

11. Castellucci P, Nanni C, Farsad M, et al. Potential pitfalls of $18 \mathrm{~F}-F D G$ PET in a large series of patients treated for malignant lymphoma: prevalence and scan interpretation. Nucl Med Commun 2005;26:689-694.

12. Petrausch U, Samaras P, Haile SR, et al. Risk-adapted FDG-PET/CT-based follow-up in patients with diffuse large B-cell lymphoma after first-line therapy. Ann Oncol 2010;21:1694-1698.

13. Cheah CY, Hofman MS, Dickinson M, et al. Limited role for surveillance PET-CT scanning in patients with diffuse large B-cell lymphoma in complete metabolic remission following primary therapy. Br J Cancer 2013;109:312-317.

14. Hamlin PA, Zelenetz AD, Kewalramani T, et al. Age-adjusted international prognostic index predicts autologous stem cell transplantation outcome for patients with relapsed or primary refractory diffuse large B-cell lymphoma. Blood 2003;102:1989-1996.

15. Swerdlow SH, Campo E, Pileri SA, et al. The 2016 revision of the World Health Organization classification of lymphoid neoplasms. Blood 2016;127:2375-2390. 
16. Lister TA, Crowther D, Sutcliffe SB, et al. Report of a committee convened to discuss the evaluation and staging of patients with Hodgkin's disease: Cotswolds meeting. J Clin Oncol 1989;7:1630-1636.

17. International Non-Hodgkin's Lymphoma Prognostic Factors Project. A predictive model for aggressive non-Hodgkin's lymphoma. N Engl J Med 1993;329:987-994.

18. Stansfeld AG, Diebold J, Noel H, et al. Updated Kiel classification for lymphomas. Lancet 1988;1:292-293.

19. Tilly H, Gomes da Silva M, Vitolo U, et al. Diffuse large B-cell lymphoma (DLBCL): ESMO Clinical Practice Guidelines for diagnosis, treatment and follow-up. Ann Oncol 2015;26 Suppl 5:v116-v125.

20. Zinzani PL, Stefoni V, Tani M, et al. Role of [18F]fluorodeoxyglucose positron emission tomography scan in the follow-up of lymphoma. J Clin Oncol 2009;27:1781-1787.
21. Mikhaeel NG, Timothy AR, O’Doherty MJ, Hain S, Maisey MN. 18-FDG-PET as a prognostic indicator in the treatment of aggressive non-Hodgkin's lymphoma-comparison with CT. Leuk Lymphoma 2000;39:543-553.

22. Meignan M, Gallamini A, Meignan M, Gallamini A, Haioun C. Report on the first international workshop on interim-PET-scan in lymphoma. Leuk Lymphoma 2009;50:1257-1260.

23. Avivi I, Zilberlicht A, Dann EJ, et al. Strikingly high false positivity of surveillance FDG-PET/CT scanning among patients with diffuse large cell lymphoma in the rituximab era. Am J Hematol 2013;88:400-405.

24. Han HS, Escalon MP, Hsiao B, Serafini A, Lossos IS. High incidence of false-positive PET scans in patients with aggressive non-Hodgkin's lymphoma treated with rituximab-containing regimens. Ann Oncol 2009;20:309-318. 